\title{
Ribonucleotide reductase M2 subunit expression and prognostic value in nasopharyngeal carcinoma
}

\author{
PING HAN ${ }^{1}$, ZHI-RUI LIN ${ }^{2}$, LI-HUA XU ${ }^{2,3}$, QIAN ZHONG ${ }^{2}$, XIAO-FEN ZHU ${ }^{4}$, FA-YA LIANG $^{1}$, \\ QIAN CAI ${ }^{1}$, XIAO-MING HUANG ${ }^{1}$ and MU-SHENG ZENG ${ }^{2}$
}

\begin{abstract}
${ }^{1}$ Department of Otolaryngology-Head and Neck Surgery, Sun Yat-sen Memorial Hospital, Sun Yat-sen University, Guangzhou, Guangdong 510120; ${ }^{2}$ Sun Yat-sen University Cancer Center, State Key Laboratory of Oncology in South China, Collaborative Innovation Center for Cancer Medicine, Guangzhou, Guangdong 510060; ${ }^{3}$ Center of Oncology and Hematology,

The First Affiliated Hospital of Guangzhou Medical University, Guangzhou, Guangdong 510120; ${ }^{4}$ Department of

Otolaryngology-Head and Neck Surgery, Huizhou First Hospital, Huizhou, Guangdong 516000, P.R. China
\end{abstract}

Received April 23, 2014; Accepted December 17, 2014

DOI: $10.3892 / \mathrm{mmr} .2015 .3360$

\begin{abstract}
The ribonucleotide reductase M2 subunit (RRM2) modulates the enzymatic activity of ribonucleotide reductase, and is involved in tumor progression. Recently, high levels of RRM2 expression were reported to correlate with poor survival outcomes in patients with colorectal and bladder cancer. However, changes in RRM2 expression in nasopharyngeal carcinoma (NPC), and its effect on the prognosis of this disease remain unknown. The aim of the present study was to analyze the expression of RRM2 in NPC cell lines, and to identify whether RRM2 may serve as a biomarker with which to assess the prognosis of NPC. The present study found that RRM2 expression was higher in NPC cell lines and tissue samples than in noncancerous nasopharyngeal epithelial cell lines and noncancerous tissues, as shown by reverse transcription-quantitative polymerase chain reaction analysis, western blotting and immunohistochemistry staining. Kaplan-Meier survival analysis demonstrated that patients with higher RRM2 expression levels had poorer disease-free survival outcomes than those with lower expression levels of RRM2. Univariate analysis showed that a lower survival rate was significantly associated with high RRM2 expression levels [hazard ratio (HR), 6.424; 95\% confidence interval $(\mathrm{CI}), 2.381-17.333 ; \mathrm{P}<0.001]$.
\end{abstract}

Correspondence to: Dr Xiao-Ming Huang, Department of Otolaryngology-Head and Neck Surgery, Sun Yat-sen Memorial Hospital, Sun Yat-sen University, 107 Yanjiang West Road, Guangzhou, Guangdong 510120, P.R. China

E-mail: xiaoming.huang@126.com

Dr Mu-Sheng Zeng, Sun Yat-sen University Cancer Center, State Key Laboratory of Oncology in South China, Collaborative Innovation Center for Cancer Medicine, 651 Dongfeng East Road, Guangzhou, Guangdong 510060, P.R. China

E-mail: zengmsh@sysucc.org.cn

Key words: ribonucleotide reductase M2, nasopharyngeal carcinoma, prognosis
Multivariate analysis indicated that RRM2 expression is an independent prognostic factor for patients with NPC (HR, 3.461; 95 \% CI, 1.204-9.949; $\mathrm{P}=0.021$ ). Overexpression of RRM2 led to increased cell proliferation, colony formation, migration and invasion in vivo. These results suggest that high levels of RRM2 expression may be a useful predictor for survival in patients with NPC and may serve as a novel prognostic indicator for these individuals.

\section{Introduction}

Nasopharyngeal carcinoma (NPC) is a type of malignant carcinoma, which is distinct in terms of its epidemiology, biological markers, clinical presentation, carcinogenic risk factors and prognostic factors. NPC is endemic to certain regions of the world, in particular in Southeast Asia, where the yearly incidence rate is 20-40/100,000 people (1). Among head and neck cancers, NPC is a type of squamous cell carcinoma, which may be highly invasive and give rise to metastases. A classification based on tumor stage, regional lymph node stage and presence or absence of metastasis (TNM classification)is usually applied to malignant tumors in order to evaluate the response to treatment and to predict prognosis (2). With the improvement of radiation techniques, the majority of patients with NPC may be cured, when diagnosed and treated in the early stages of the disease (3). However, in the advanced stages of the disease, distant metastases usually result in treatment failure (4). Nevertheless, studies have shown that even patients with the same stage of NPC exhibit marked variations in their response to similar treatments, indicating that the current staging system remains inadequate for making accurate predictions $(5,6)$. Therefore, novel NPC biomarkers may be useful for assessing the prognosis of the disease and may facilitate the development of personalized therapies for patients with $\operatorname{NPC}(7,8)$.

Ribonucleotide reductase (RR) is a enzyme involved in the DNA synthesis pathway (9). RR is responsible for the conversion of ribonucleoside diphosphate to deoxyribonucleoside diphosphate, which is essential for DNA synthesis and repair (10). Human RR consists of two subunits, M1 and M2. RR enzymatic activity is controlled by its M2 subunit 
(RRM2) (11). It has been reported that RRM2 is physiologically associated with DNA synthesis and cell proliferation. Changes in RRM2 expression and its potential prognostic value have been demonstrated in a variety of human malignancies, including bladder cancer (12), esophageal carcinoma (13), melanoma (14) and gastric carcinoma (15). Thus, RRM2 is hypothesized to be involved in malignant progression (16). To date, however, little is known regarding the expression of RRM2 and its prognostic value and function in NPC.

Therefore, the present study aimed to investigate RRM2 expression in NPC, in order to identify whether RRM2 may be a prognostic biomarker for patients with NPC. The present study also aimed to explore whether RRM2 may influence the biological behavior of NPC cells in vitro.

\section{Materials and methods}

Tissue specimens. The first set of samples consisted of 60 NPC tissue samples and 20 noncancerous nasopharyngeal epithelial tissue samples. Cancerous and noncancerous samples were collected from Sun Yat-sen University CancerCenter(SYSUCC) and Sun Yat-sen Memorial hospital (Guangzhou, China), respectively, between January and October 2011. These samples were used to investigate RRM2 expression using reverse transcription-quantitative polymerase chain reaction (RT-qPCR). Samples were immediately immersed into an RNAlater solution (AM7021, Ambion Life Technologies, Carlsbad, CA, USA) and incubated overnight at $4^{\circ} \mathrm{C}$, followed by incubation at $-80^{\circ} \mathrm{C}$ prior to RNA extraction. The second set of samples consisted of 56 paraffin-embedded NPC tissue samples and four noncancerous nasopharyngeal epithelial tissue samples, collected from Huizhou Municipal Central Hospital (Huizhou, China), between January 2002 and March 2006. These samples were used to conduct an immunohistochemistry (IHC) assay. The clinicopathological characteristics of the 56 patients with NPC are summarized in Table I. All patients had been treated with standard curative radiotherapy, with $(n=16)$ or without $(n=40)$ chemotherapy. The TNM stage for the 56 patients was defined according to the International Union against Cancer (UICC) 2002 guidelines.

Written informed patient consent and ethical approval from the Sun Yat-sen Memorial hospital, SYSUCC and Huizhou Municipal Central Hospital Institute Research Ethics Committee were obtained in order to use these clinical materials for research purposes.

The follow-up time was calculated from the first day of treatment to either the day the patient was deceased or the day of the last examination. Patients were examined at least every three months during the first two years of treatment and thereafter, every six months until the end-of-life or the end of the study. The date of the last follow-up was March 2011. The median follow-up time was 51 months (3-83 months). The time to the primary end-points was assessed as overall survival (OS) and distant metastasis-free survival (DMFS).

IHC assay. NPC tissue samples and noncancerous nasopharyngeal epithelial tissue samples were resected from patients, fixed in formalin and embedded in paraffin. Following this, the tissue samples were cut into $4-\mu \mathrm{m}$ sections and heated at $58^{\circ} \mathrm{C}$ for $3 \mathrm{~h}$. The sections were dewaxed in
Table I. Characteristics of 56 patients with NPC.

\begin{tabular}{|c|c|}
\hline Characteristic & Cases \\
\hline \multicolumn{2}{|l|}{ Age (years) } \\
\hline Median, range & $46,20-78$ \\
\hline$<46$ & $22(39.3 \%)$ \\
\hline$\geq 46$ & $34(60.7 \%)$ \\
\hline \multicolumn{2}{|c|}{ Follow-up period (months) } \\
\hline Median, range & $51,2-83$ \\
\hline \multicolumn{2}{|l|}{ Gender } \\
\hline Male & $45(80.3 \%)$ \\
\hline Female & $11(19.6 \%)$ \\
\hline \multicolumn{2}{|l|}{ T stage } \\
\hline $\mathrm{T} 1$ & $7(12.5 \%)$ \\
\hline $\mathrm{T} 2$ & $18(32.2 \%)$ \\
\hline T3 & $21(37.5 \%)$ \\
\hline $\mathrm{T} 4$ & $10(17.8 \%)$ \\
\hline \multicolumn{2}{|l|}{$\mathrm{N}$ stage } \\
\hline N0 & $8(14.3 \%)$ \\
\hline N1 & $18(32.2 \%)$ \\
\hline $\mathrm{N} 2$ & $23(41.1 \%)$ \\
\hline N3 & $7(12.5 \%)$ \\
\hline \multicolumn{2}{|l|}{ UICC stage } \\
\hline I & $3(5.4 \%)$ \\
\hline II & $13(23.2 \%)$ \\
\hline III & $24(43.0 \%)$ \\
\hline IV & $16(28.6 \%)$ \\
\hline \multicolumn{2}{|l|}{ WHO type } \\
\hline NKUC & $54(96.4 \%)$ \\
\hline NKDC & $2(3.6 \%)$ \\
\hline \multicolumn{2}{|l|}{ Metastasis } \\
\hline Yes & $10(17.9 \%)$ \\
\hline No & $46(82.15 \%)$ \\
\hline \multicolumn{2}{|l|}{ Survival } \\
\hline Yes & $29(51.8 \%)$ \\
\hline No & $27(48.2 \%)$ \\
\hline \multicolumn{2}{|l|}{ Chemotherapy } \\
\hline Yes & $16(28.5 \%)$ \\
\hline No & $40(71.5 \%)$ \\
\hline
\end{tabular}

NPC, nasopharyngeal carcinoma; NKUC, non-keratinizing undifferentiated carcinoma; NKDC; non-keratinizing differentiated carcinoma; UICC, international union against cancer; T stage, tumor stage; N stage, regional lymph node stage; WHO, world health organization.

xylene and rehydrated through graded alcohol to distilled water. The sections were then immersed in 3\% hydrogen peroxide for $15 \mathrm{~min}$ at room temperature, in order to block endogenous peroxidase activity. The sections were heated in an antigen retrieval solution (citrate, $\mathrm{pH}$ 6.0; ZSGB-BIO, Beijing, China) for $4 \mathrm{~min}$ in a pressure cooker at $150^{\circ} \mathrm{C}$. Once the retrieval solution had cooled to room temperature (for $2 \mathrm{~h}$ 
to reach approximately $26^{\circ} \mathrm{C}$ ), the sections were incubated either with diluted mouse monoclonal anti-RRM2 antibody (1:200; NBP1-69832; Novus Biologicals, Littleton, CO, USA) or with the negative control (mouse immunoglobulin G; Abcam, Shanghai, China), overnight at $4^{\circ} \mathrm{C}$. The sections were then washed with phosphate-buffered saline Tween-20 (PBST; ZSGB-BIO) three times and incubated with the goat anti-mouse immunoglobulin-G-horseradish peroxidase secondary antibodies (ADR-5307; ZSGV-BIO) for $30 \mathrm{~min}$ at $37^{\circ} \mathrm{C}$. They were washed with PBST three times, followed by 3,3-diaminobenzidine (DAB) staining for $2 \mathrm{~min}$ in order to visualize the target protein (RRM2). Sections were then counter-stained with hematoxylin in order to visualize the nucleus. They were then washed with running water for $2 \mathrm{~h}$ and dehydrated at $37^{\circ} \mathrm{C}$. Finally, the sections were preserved in neutral balsam (ZSGB-BIO).

Measuring RRM2 expression using an IHC assay. All sections were stained using DAB (ZSGB-BIO) for 5 min each. For each slide, five random fields of vision were selected for scoring and the mean score for each slide was used for the final analysis. Positive staining was scored as follows: 0 , negative or $<10 \%$ positive cancer cells; $1,10-25 \%$ positive cancer cells; $2,26-60 \%$ positive cancer cells; and 3, $>60 \%$ positive cancer cells. Staining intensity was categorized as follows: 0 , no staining; 1, weak staining (light yellow); 2, moderate staining (yellow-brown); and 3, strong staining (brown). The scoring was confirmed by at least two pathologists in a double-blind model analysis. The RRM2 expression immunoreactivity score (IRS) was calculated as follows: IRS = intensity score $\mathrm{x}$ positive staining score. An optimal cut-off value for high and low RRM2 expression was based on a measurement of heterogeneity with a log-rank test statistical analysis regarding the overall survival (OS). IRS $\leq 5.0$ indicated a low RRM2 expression and IRS >5.0 indicated a high RRM2 expression.

Cell lines and cell cultures. Two nasopharyngeal epithelial cell lines (NPECs) were immortalized by transfection with B lymphoma Mo-MLV insertion region 1 (Bmi1) homolog, which was synthesized by Shanghai Invitrogen Shanghai Biotechnology Co., Ltd (Guangzhou, China), as described previously $(17,18)$ and cultured in a keratinocyte serum-free medium (Invitrogen Life Technologies, Carlsbad, CA, USA). The two immortalized NPEC cells lines were referred to as NPEC1-Bmil and NPEC2-Bmi1. NPECs N01-N09 were derived from a primary culture of fresh nasopharyngeal tissues. The following NPC cell lines were cultured in an RPMI-1640 medium (Gibco-BRL Life Technologies, Carlsbad, USA) with $10 \%$ fetal bovine serum (FBS; Gibco-BRL Life Technologies): CNE1, CNE2, 6-10B, 5-8F, HONE1, SUNE1 and HNE1. NPECs and NPC cell lines were cultured at $37^{\circ} \mathrm{C}$ with $5 \% \mathrm{CO}_{2}$.

$R N A$ extraction and $R T-q P C R$ analysis. Total RNA from the NPECs and NPC cell lines, and from human NPC tissue samples and noncancerous nasopharyngeal epithelial tissue samples, was extracted using TRIzol reagent (Invitrogen Life Technologies). RNA concentrations and quantities were determined using a NanoDrop spectrophotometer (ND-1000, Thermo Fisher Scientific, Rockford, IL, USA). Following reverse transcription of the total RNA, the first-strand cDNA was used as a template for measuring RRM2 expression. RT-qPCR and data collection were conducted using a CFX96 real-time PCR detection system (Bio-Rad, Hercules, CA, USA). The RT-qPCR detection was normalizedusing aninternalcontrol(Glyceraldehyde-3-phosphate dehydrogenase; Gapdh). The following primers were used: Forward: 5'-TTGGGATGAATTGCACTCTAA-3', and reverse: 5'-CTGATACTCGCCTACTCT-3' for RRM2, and forward: 5'- CTCCTCCTGTTCGACAGTCAGC-3' and reverse: 5'-CCCAATACGACCAAATCCGTT-3' for Gapdh. In order to ensure that the results were reproducible, all experiments were repeated three times.

Western blotting. Protein from NPEC1-Bmil and from the following NPC cell lines: CNE1, CNE2, HONE1, HNE1, 6-10B, C666, SUNE1 and 5-8F, was extracted according to the method described by Liao et al (19). Western blot analysis was performed according to the method described by Sun et al (20). The proteins of all cell lines were incubated overnight at $4^{\circ} \mathrm{C}$ with a primary rabbit polyclonal antibody against human RRM2 (1:500 dilution; NBP-69832; Novus Biologicals). In order to confirm equal loading of the samples, the membranes were stripped and re-probed with a mouse monoclonal antibody against human Gapdh (1:4,000 dilution; sc-137179; Santa Cruz Biotechnology, Inc., Santa Cruz, CA, USA). Three independent experiments were conducted for each assay.

Plasmids and transient transfection. The full-length human RRM2 was cloned into a pcDNA3.1 vector (Shanghai Invitrogen Biotechnology Co., Ltd) using the restriction enzymes Bam HI and Xho I(New England Biolabs, Inc.,Ipswich,MA,USA). HNE1 cells $\left(3.0 \times 10^{5}\right)$ were seeded in six-well culture plates. When the cells had reached $60 \%$ confluence, they were transfected with either a pcDNA3.1-vector or a pcDNA3.1-RRM2 plasmid using Lipofectamine $2000^{\circledR}$ (Invitrogen Life Technologies) in order to overexpress RRM2. Following a $6 \mathrm{~h}$ incubation period at $37^{\circ} \mathrm{C}$ in $5 \% \mathrm{CO}_{2}$, the transfection medium was replaced with $2 \mathrm{ml}$ fresh culture medium. Following $36 \mathrm{~h}$ of transfection, the cells were collected for western blotting, RT-qPCR, cell proliferation, colony formation, invasion and migration assays.

Cell proliferation assay. A 3-(4,5-dimethylthiazol-2-yl)-2,5diphenyltetrazoliumbromide (MTT) assay was conducted in order to investigate cell proliferation. HNE1-pcDNA3.1-vector (control cells) and HNE1-pcDNA3.1-RRM2 (cells overexpressing RRM2) were incubated in 96-well plates at an equal initial cell density of $1 \times 10^{3}$. MTT was diluted to $5 \mathrm{mg} / \mathrm{ml}$ with phosphate-buffered saline, and the MTT solution (20 $\mu \mathrm{l})$ was added to the supernatant in each well. The 96-well plates were then incubated at $37^{\circ} \mathrm{C}$ for $4 \mathrm{~h}$. Subsequently, dimethylsulfoxide solution (200 $\mu$; ; Sigma-Aldrich, St. Louis, MO, USA) was added to the wells in order to melt the sediment. The plates were then shaken for $10 \mathrm{~min}$ at $106 \mathrm{x} \mathrm{g}$ in order to mix the solutions. At $24 \mathrm{~h}$ intervals, the optical density (OD) value was detected with a microplate reader (Molecular Devices, Sunnyvale, CA, USA). Three independent experiments were conducted for each assay.

Colony formation assay. Three sets of 200 HNE1-pcDNA3.1-vector (control cells) and three sets of 200 
A

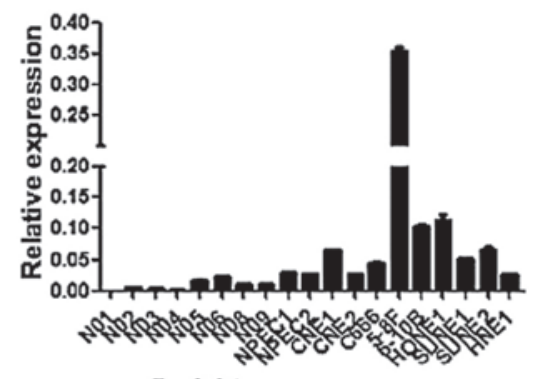

B

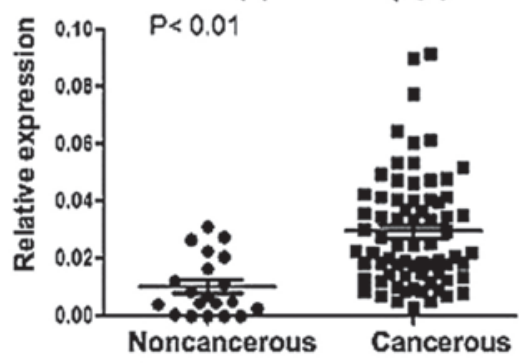

C

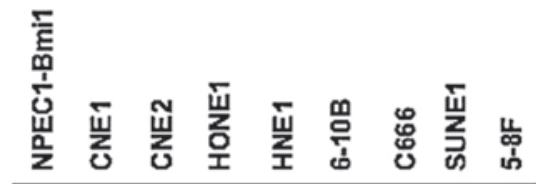

Anti-RRM2

Anti-Gapdh

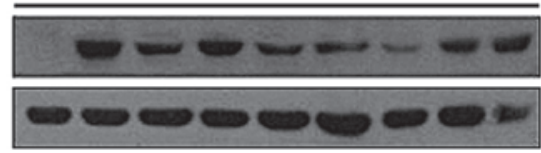

Figure 1. RRM2 expression in cell lines and nasopharyngeal tissues. (A) RRM2 mRNA expression level in the NPC cell lines was higher than that of the NPECs and the two immortalized NPECs. Three independent experiments were performed. (B) RRM2 expression was higher in the NPC cell lines compared with the NPEC1-Bmil cell line. (C) RRM2 mRNA expression levels in 60 NPC specimens and 20 noncancerous nasopharyngeal epithelia tissues were determined using reverse transcription-quantitative polymerase chain reaction $(\mathrm{P}<0.01)$. RRM2, ribonucleotide reductase M2 subunit NPEC, nasopharyngeal epithelial cell lines; NPC, nasopharyngeal carcinoma; Gapdh, Glyceraldehyde 3-phosphate dehydrogenase.

HNE1-pcDNA3.1-RRM2 (cells overexpressing RRM2) were plated at 200 cells per well in six-well plates and cultured and incubated for 10 days at $37^{\circ} \mathrm{C}$. Once the majority of the colonies had grown to $>50$ cells, the cells were washed and fixed in methanol for $15 \mathrm{~min}$. Subsequently, the cells were dyed with crystal violet (Sigma-Aldrich) for $15 \mathrm{~min}$ at room temperature. Three independent experiments were conducted for each assay.

Wound-healing assay. A wound-healing assay was conducted in order to determine the rate of migration for the control cells and cells overexpressing RRM2, according to the method described by Song et al (18). Three independent experiments were conducted for each assay.

Transwell assay. A transwell assay was conducted in order to determine the invasive ability of control cells and cells overexpressing RRM2, according to the method described by Song et al (18). Briefly, the upper chambers with an $8 \mathrm{mM}$ porosity polycarbonate membrane (BD Biosciences, Franklin Lakes, NJ, USA) were pre-coated with Matrigel ${ }^{\mathrm{TM}}$ (BD Biosciences) and covered with $200 \mu \mathrm{l}$ of medium without FBS, which contained $1 \times 10^{5}$ cells. The chambers were then immersed in the lower wells, which contained $500 \mu \mathrm{l}$ of medium with $10 \%$ FBS. Cells which traversed the filters and adhered to the opposite side of the chamber membrane were photographed and counted by light microscopy (IX71; Olympus Corp., Tokyo, Japan) after $24 \mathrm{~h}$. Three independent experiments were conducted for each assay.

Statistical analysis. SPSS 16.0 (SPSS, Inc., Chicago, IL, USA) was used for statistical analyses. The correlation between RRM2 level and clinicopathological features of the patients were analyzed using either a $\chi^{2}$ test or Fischer's exact test. Differences among variables were assessed using 2-tailed Student's t-test. Survival curves were plotted using Kaplan-Meier survival analysis and compared using a log-rank test. Univariate and multivariate regression analyses were performed using the Cox proportional hazards regression model to determine the effect of particular prognostic factors on OS. In all cases $\mathrm{P}<0.05$ was considered to indicate a statistically significant difference.

\section{Results}

Expression of RRM2 in cell lines and tissue samples. In order to measure RRM2 expression, RT-qPCR was conducted for the following NPECs: NPEC1-Bmil and NPEC2-Bmi1, and for the following NPC cell lines: CNE1, CNE2, 6-10B, 5-8F, HONE1, SUNE1 and HNE1. RRM2 mRNA expression level was higher in NPC cell lines compared with the NPECs, in particular in the NPEC cell line, $5-8 \mathrm{~F}$ (Fig. 1A). The RRM2 protein expression of these cell lines were assessed using western blotting. As NPEC1 and NPEC2 showed similar mRNA expression levels of RRM2, RRM2 protein expression was only measured in the NPEC1-Bmil cell line, where it was not found to be expressed. However RRM2 protein expression was high in the majority of the NPC cell lines (Fig. 1B). In order to further examine whether there was high RRM2 expression in tissue samples from patients with NPC compared with that in healthy tissue samples, an RT-qPCR analysis was conducted for 60 NPC tissue samples and 20 noncancerous nasopharyngeal epithelial samples. The mean RRM2 expression level in NPC tissue samples was significantly higher than in noncancerous nasopharyngeal epithelial tissue samples (Fig. 1C). In conclusion, RRM2 expression was upregulated in NPC cell lines and tissue samples, compared with that in NPECs and noncancerous tissue samples.

RRM2 expression is upregulated in NPC tissue samples. In order to examine the expression of the RRM2 protein in NPC tissue samples, an IHC analysis was conducted for 56 NPC specimens and four noncancerous nasopharyngeal epithelial tissues, using a monoclonal antibody. No staining was observed in the four noncancerous nasopharyngeal epithelial tissue samples (Fig. 2A). Among the 56 NPC tissue samples, four (7.1\%) showed negative staining (Fig. 2A), 16 (28.6\%) showed weak staining (Fig. 2B), 20 (35.7\%) showed moderate staining (Fig. 2C), and 16 (28.6\%) showed strong staining (Fig. 2D). Furthermore, RRM2 expression was predominantly localized to the cytoplasm of NPC cells (Fig. 2).

Association of RRM2 expression with clinicopathological characteristics. The association between RRM2 expression in 
Table II. Correlation between RRM2 expression and clinicopathologic characteristics in NPC patients.

\begin{tabular}{|c|c|c|c|c|}
\hline \multirow[b]{2}{*}{ Characteristic } & \multirow{2}{*}{$\begin{array}{c}\text { All cases } \\
(\mathrm{n}=56)\end{array}$} & \multicolumn{2}{|c|}{ RRM2 expression $(n=56)$} & \multirow[b]{2}{*}{ P-value } \\
\hline & & Low $(\mathrm{n}=24 ; \%)$ & High $(\mathrm{n}=32 ; \%)$ & \\
\hline Age (years) & & & & 0.178 \\
\hline$<46$ & 22 & $12(50.0)$ & $10(31.2)$ & \\
\hline$\geq 46$ & 34 & $12(50.0)$ & $22(68.8)$ & \\
\hline Gender & & & & 0.113 \\
\hline Male & 45 & $17(70.8)$ & $28(87.5)$ & \\
\hline Female & 11 & 7 (29.2) & $4(12.5)$ & \\
\hline T stage & & & & $0.001^{\mathrm{a}}$ \\
\hline T1-2 & 25 & $17(70.8)$ & $8(25.0)$ & \\
\hline T3-4 & 31 & 7 (29.2) & $24(75.0)$ & \\
\hline $\mathrm{N}$ stage & & & & $0.034^{\mathrm{a}}$ \\
\hline N0-1 & 26 & $15(62.5)$ & $11(34.4)$ & \\
\hline $\mathrm{N} 2-3$ & 30 & 9 (37.5) & $21(65.6)$ & \\
\hline UICC stage & & & & $0.000^{\mathrm{a}}$ \\
\hline I-II & 16 & $13(54.2)$ & $3(9.4)$ & \\
\hline III-IV & 40 & $11(45.8)$ & $29(90.6)$ & \\
\hline WHO type & & & & 0.678 \\
\hline NKUC & 54 & $23(95.8)$ & $31(96.9)$ & \\
\hline NKDC & 2 & $1(4.2)$ & $1(3.1)$ & \\
\hline Metastasis & & & & 0.064 \\
\hline Yes & 10 & $2(8.3)$ & $7(21.9)$ & \\
\hline No & 46 & $22(91.7)$ & $25(78.1)$ & \\
\hline Survival state & & & & $0.000^{\mathrm{a}}$ \\
\hline Survival & 29 & $19(79.2)$ & $10(31.2)$ & \\
\hline Mortality & 27 & $5(20.8)$ & $22(68.8)$ & \\
\hline
\end{tabular}

NKUC, non-keratinizing undifferentiated carcinoma; NKDC, non-keratinizing differentiated carcinoma; RRM2, ribonucleotide reductase M2 subunit; NPC, nasopharyngeal carcinoma; UICC, international union against cancer; WHO, world health organization; T stage, tumor stage; $\mathrm{N}$ stage, nearby regional lymph nodes.

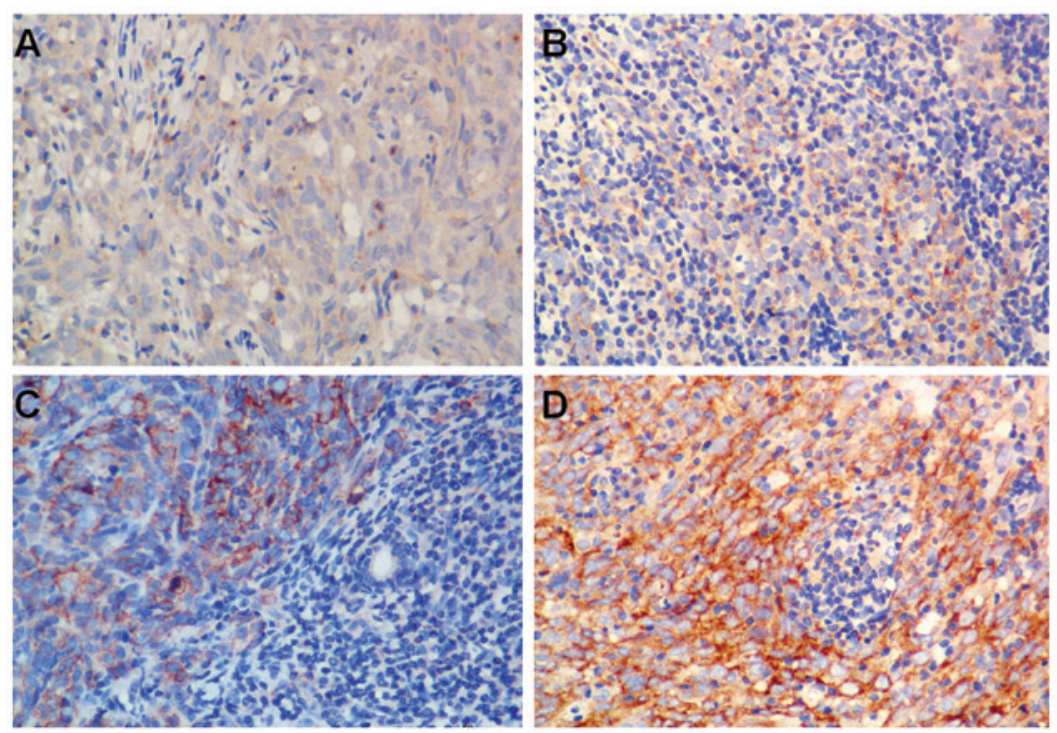

Figure 2. Measurement of RRM2 protein expression using immunohistochemistry analysis in nasopharyngeal carcinoma tissues. RRM2 protein expression was primarily localized in the cytoplasm. Representative images of nasopharyngeal carcinoma grading: (A) Negative control, not expressing RRM2 (x400; $n=4)$; (B) weak staining ( $x 400 ; n=16)$; (C) moderate staining ( $x 400 ; n=20)$; and (D) strong staining $(x 400 ; n=16)$. RRM2, ribonucleotide reductase M2 subunit. 
Table III. Univariate and multivariate Cox regression analyses of prognostic variables in 56 patients with NPC.

\begin{tabular}{lccc}
\hline Variable & Subset & HR $(95 \%$ CI $)$ & P-value \\
\hline Univariate analysis & & & \\
RRM2 expression & High vs Low & $6.424(2.381-17.333)$ & $<0.001^{\mathrm{a}}$ \\
Age (years) & $>$ 46 vs $\leq 46$ & $5.71(1.793-16.608)$ & $0.001^{\mathrm{a}}$ \\
Gender & Female vs Male & $0.539(0.162-1.794)$ & 0.314 \\
T staging & T1-2 vs T3-4 & $11.214(3.289-38.227)$ & $<0.001^{\mathrm{a}}$ \\
N staging & N0-1 vs N2-3 & $0.125(0.844-4.061)$ & 0.125 \\
Metastasis & Yes vs No & $3.216(1.336-7.744)$ & $0.009^{\mathrm{a}}$ \\
UICC stage & I -II vs III -IV & $8.247(1.913-35.555)$ & $0.005^{\mathrm{a}}$ \\
WHO histological classification & NKDC vs NKUC & $2.147(0.505-9.126)$ & 0.301 \\
Multivariate analysis & & & $<0.001^{\mathrm{a}}$ \\
T staging & T1-2 vs T3-4 & $11.214(3.289-38.227)$ & $0.021^{\mathrm{a}}$ \\
RRM2 expression & High vs Low & $3.461(1.204-9.949)$ & $0.026^{\mathrm{a}}$ \\
Age (years) & $>46$ vs $\leq 46$ & $4.087(1.233-13.545)$ & $0.041^{\mathrm{a}}$ \\
UICC stage & I -II vs III -IV & $0.623(0.049-17.972)$ &
\end{tabular}

HR, hazard ratio; RRM2, ribonucleotide reductase M2 subunit; NPC, nasopharyngeal carcinoma; UICC, international union against cancer; WHO, world health organization; NKUC, non-keratinizing undifferentiated carcinoma; NKDC, non-keratinizing differentiated carcinoma T stage, tumor stage; $\mathrm{N}$ stage, nearby regional lymph nodes.
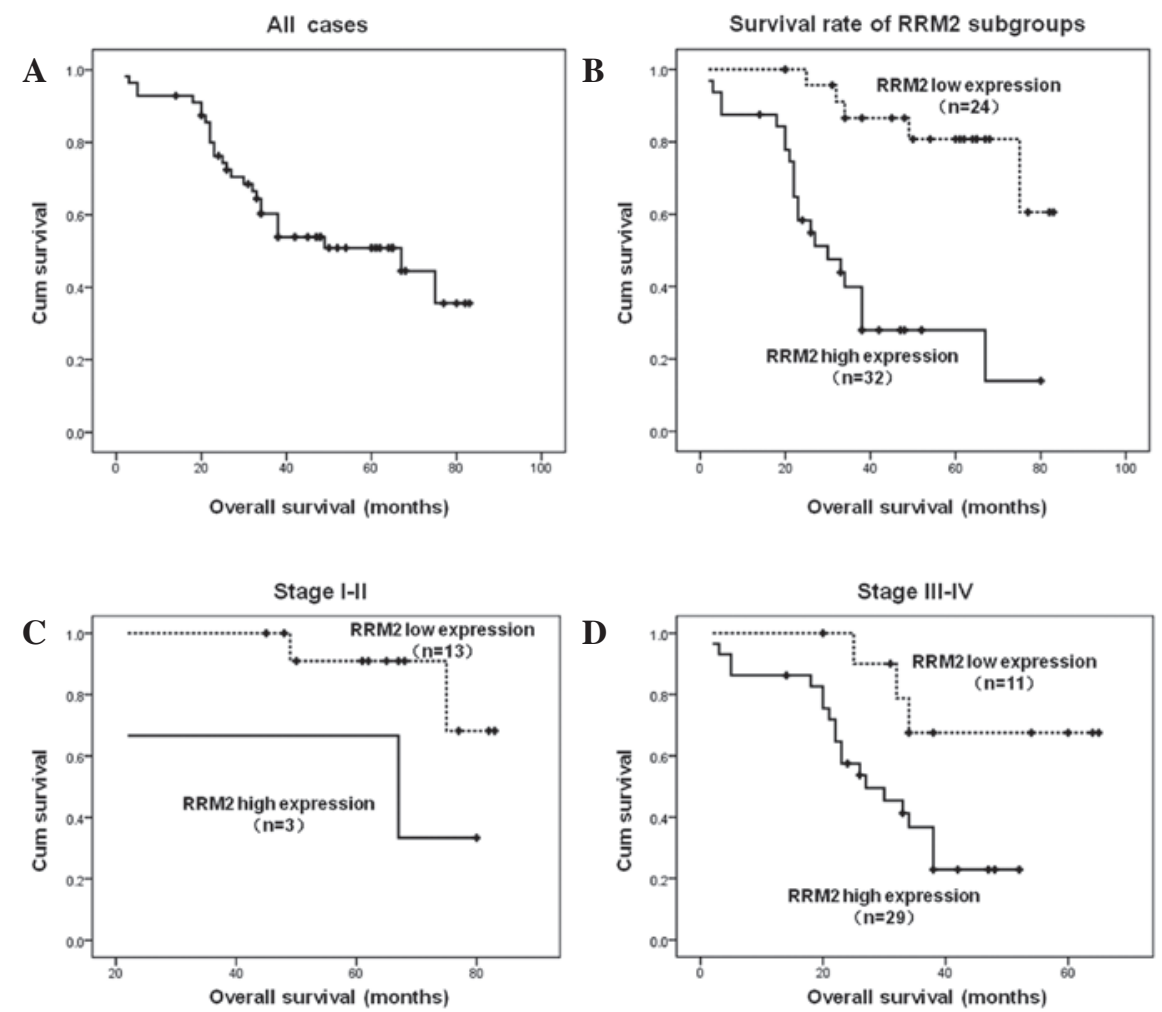

Figure 3. Correlation between RRM2 expression and NPC patient survival using Kaplan-Meier survival curve and log-rank test analyses. (A) Of 56 patients with NPC, 51.8\% had an OS of five years. (B) High RRM2 expression level was significantly correlated with OS (P<0.05) in patients with NPC. (C) A high RRM2 expression level was significantly correlated with OS $(\mathrm{P}<0.001)$ in patients with an early stage of NPC (UICC stage I-II). (D) A high RRM2 expression level was significantly associated with OS $(\mathrm{P}<0.05)$ in patients with an advanced stage of NPC (UICC stage III-IV). RRM2, ribonucleotide reductase M2 subunit; NPC, nasopharyngeal carcinoma; OS, Overall survival; Cum, cumulative; UICC, international union against cancer.

NPC tissue samples and the clinicopathological characteristics of patients with NPC was analyzed. Samples were separated into two groups with low or high RRM2 expression, according a cutoff score of 5.0 (21). Tissue samples of patients with NPC, which had a score of $>5.0$ were defined as having high RRM2 expression, while a score $\leq 5.0$ was defined as low 
A

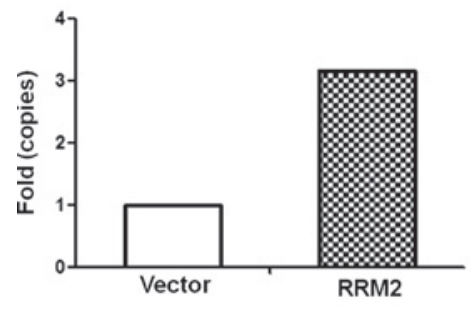

B

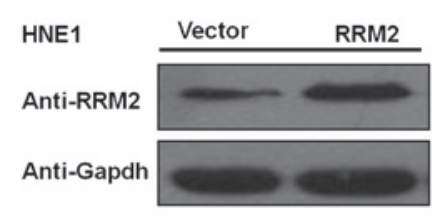

C

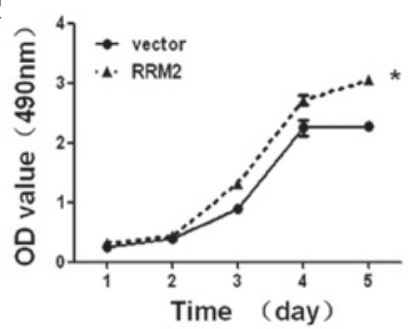

D
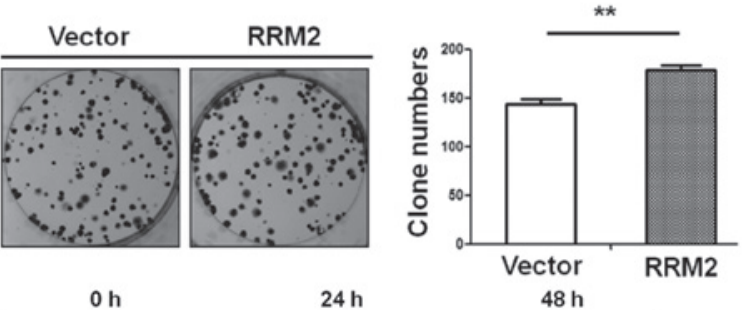

E

o h

$24 \mathrm{~h}$
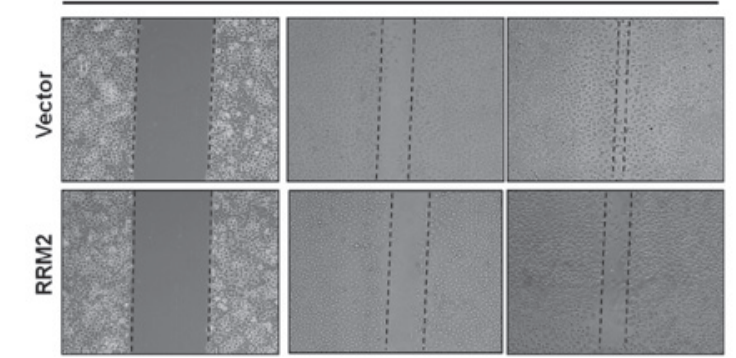

F
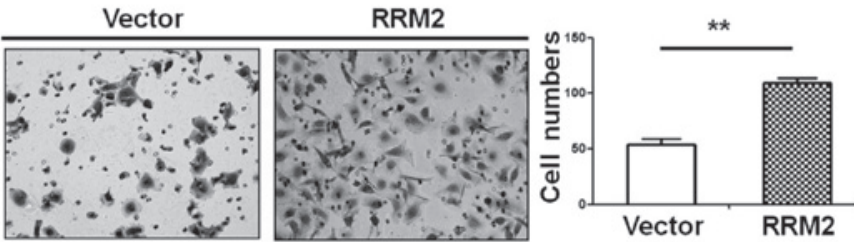

Figure 4. RRM2 overexpression enhanced NPC cell proliferation, colony formation, migration and invasion capability. All data are expressed as the mean \pm standard deviation. (A) Overexpression of RRM2 in HNE1 cells at $36 \mathrm{~h}$ post-transfection. HNE1 cells were transfected either with the pcDNA3.1-RRM2 vector or the control plasmid pcDNA3.1 vector. RRM2 mRNA levels increased in HNE1-pcDNA3.1-RRM2 cells according to real time quantitative-polymerase chain reaction. (B) Western blot analysis showed the RRM2 protein level expression in cells overexpressing RRM2 and control cells following transfection. (C) Growth curves of the cells overexpressing RRM2 and control cells. Following RRM2 transient transfection, the cells overexpressing RRM2 grew significantly faster than the control cells during days 3-5 ("P<0.05). (D) Colony formation assay of the cells overexpressing RRM2 and control cells. Cells overexpressing RRM2 formed more colonies compared with the control cells ( $\left.{ }^{* *} \mathrm{P}<0.01\right)$. (E) Wound healing assay of the cells overexpressing RRM2 and control cells. Cells overexpressing RRM2 exhibited a greater wound healing response compared with the control cells. (F) Transwell migration assay of the cells overexpressing RRM2 and control cells. The migration capability was higher in the cells overexpressing RRM2 compared with the control cells $\left({ }^{* *} \mathrm{P}<0.01\right)$. RRM2, ribonucleotide reductase M2 subunit; NPC, nasopharyngeal carcinoma; Gapdh, Glyceraldehyde 3-phosphate dehydrogenase.

RRM2 expression. High RRM2 expression was observed in 32/56 (57.1\%) of NPC tissue samples. There were significant correlations between RRM2 expression and T (tumor) stage $(\mathrm{P}=0.008)$, between $\mathrm{RRM} 2$ expression and $\mathrm{N}$ (nearby regional lymph node) stage $(\mathrm{P}=0.036)$, and between RRM2 expression and UICC stage $(\mathrm{P}=0.002)$ in patients with NPC. However, there was no significant correlation between RRM2 expression and other clinicopathological features, including patient age, gender, metastasis and world health organization (WHO) classification ( $\mathrm{P}>0.05$; Table II).

Upregulation of RRM2 expression is associated with poor prognosis. Of the 56 patients with NPC assessed, $51.8 \%$ had an OS of five years (Fig. 3A). NPC tissue samples from 24 (42.9\%) patients exhibited low RRM2 expression levels while 32 (57.1\%) exhibited high RRM2 expression levels. The prognostic role of RRM2 was measured using Kaplan-Meier analysis and a log-rank test analysis in order to estimate OS in patients with NPC. The median follow-up time was 51 months. The cumulative five-year survival was $37.3 \%$ [95\% confidence interval (CI), 28.074-46.521] in the high RRM2 expression group and 72.3\% (95\% CI, 64.154-80.539) in the low RRM2 expression group ( $\mathrm{P}<0.01$; Fig. 3B). Furthermore, the patients were divided into two groups according to their UICC stage: early (I-II) and advanced (III -IV). These groups exhibited a five-year OS of 73.8\% (95\% CI: 65.257-82.330) and $38.6 \%$ (95\% CI: 31.653-45.532) respectively. The results demonstrated that the association between a high RRM2 expression and shorter OS was significantly stronger in patients with advanced disease than in those with disease at an early stages. For patients with the disease at earlier stages, the five-year OS rate was 56.3\% (95\% CI: 28.212-84.455) for patients with high expression of RRM2 and 78.1\% (95\% CI: 71.840-84.342) for patients with low expression of this gene $(\mathrm{P}<0.05)$ (Fig. 3C). For patients with an advanced stage of NPC, the five-year OS rate was $30.0 \%$ (95\% CI, 24.169-35.836) for patients with high RRM2 expression levels and 53.8\% (95\% CI, 43.303-64.297) for patients with low RRM2 expression levels $(\mathrm{P}<0.05)$ (Fig. 3D). 
High RRM2 expression is an independent prognostic factor. Univariate Cox regression analyses showed that the following characteristics were significantly correlated with five-year OS: RRM2 expression $(\mathrm{P}<0.001)$, age $(\mathrm{P}=0.001)$, $\mathrm{T}$ stage $(\mathrm{P}<0.001)$, metastasis $(\mathrm{P}=0.009)$ and UICC stage $(\mathrm{P}=0.005)$ (Table III). By contrast gender, $\mathrm{N}$ stage, and WHO histological classification exhibited no significant correlation with DMFS or OS $(\mathrm{P}>0.05)$. Multivariate analyses were conducted in order to determine whether RRM2 expression level was an independent prognostic factor in DFS and DMFS (Table III). As a result, RRM2 appeared to be an independent and unfavorable factor (hazard ratio [HR],3.461; 95\% CI, 1.204-9.949; $\mathrm{P}=0.021$ ). In addition, patient age (HR, 4.087; 95\% CI, 1.233-13.545; $\mathrm{P}=0.026)$ and T stage (HR, 11.214; 95\% CI, 3.289-38.227; $\mathrm{P}<0.001$ ), were defined as independent prognostic predictors for five-year OS (Table III). These results indicated that RRM2 is an independent prognostic factor for DMFS and OS in patients with NPC.

RRM2 accelerates the proliferation, colony formation, migration and invasiveness of NPC cells. The NPC cell line, HNE1, exhibited endogenous RRM2 expression levels (Fig. 1A). This cell line was therefore used to establish an RRM2 overexpressing cell line. Following $36 \mathrm{~h}$ transfection, RRM 2 mRNA and protein expression levels were higher in cells overexpressing RRM2 compared with control cells (Fig. 4A and B).

In order to investigate the effect of RRM2 expression on the HNE1 cells, the following analyses were performed for the RRM2 overexpressing cell line and the control cell line: AnMTT assay, a colony formation analysis, a wound healing assay and a migration assay. RRM2 overexpressing cell lines grew significantly faster than control cells $(\mathrm{P}<0.05$; Fig. 4C), suggesting that RRM2 overexpression enhanced NPC cell proliferation. Cells overexpressing RRM2 formed significantly more colonies than the control cells $(\mathrm{P}<0.01$; Fig. 4D). In addition, cells overexpressing RRM2 exhibited a more rapid wound healing response ( $\sim 8 \mathrm{~h}$ following serum starvation), compared with that of the control cells $(\mathrm{P}<0.05$, Fig. $4 \mathrm{E})$. Cells overexpressing RRM2 also exhibited a significantly faster cell invasion through the matrigel-covered chamber transwell compared with the control cells $(\mathrm{P}<0.01$, Fig. 4F $)$.

\section{Discussion}

NPC, an endemic cancer in southern China, invades into the surrounding tissues and spreads to the regional lymph nodes and distant organs. The majority of patients with NPC are diagnosed when the tumor is at an advanced stage (22). The presence of distant metastases, usually results in treatment failure (4). Radiotherapy and chemoradiotherapy may improve OS for patients with NPC. However, the prognosis remains poor in patients with advanced NPC (23-25). For the majority of patients with NPC, the TNM staging system is inadequate for providing an accurate prognosis $(26,27)$, and biomarkers may add prognostic value to the staging system. Therefore, novel molecular biomarkers are required in order to improve the accuracy of prognostication for patients with NPC.

Ribonucleotide reductase consists of two subunits: M1 and M2. RRM1 functions as a metastasis-suppressor gene through the induction of phosphatase and tensin homolog expression $(28,29)$. Ribonucleotide reductase enzymatic activity is primarily modulated by its M2 subunit (RRM2). RRM2 exhibits a role in cell division, proliferation and differentiation (30-32). Studies have shown that RRM2 influences the metastasis potential and drug-resistance of malignant cancer cells $(12,13,15,16)$. Higher RRM2 expression has been observed in several types of cancer cells, compared with that in noncancerous cells $(12,13,15)$. RRM2 expression has also been associated with tumor invasion, metastasis and the OS of cancer patients in previous studies, including, bladder and gastric cancer patients (12-16). However, the pattern of RRM2 expression and its function in NPC remain unknown.

To the best of our knowledge, the present study was the first to demonstrate that NPC tissue samples and cell lines exhibit higher RRM2 expression levels than those in noncancerous tissue samples and cell lines (Fig. 1). RRM2 overexpression induced cell growth and increased the colony formation ability of NPC cells in vitro (Fig. 4A and B), suggesting that RRM2 may be involved in NPC progression. A high RRM2 expression level was significantly correlated with the $\mathrm{T}$ stage, $\mathrm{N}$ stage and UICC stage in patients with NPC. It was also associated with low OS, in particular in patients with advanced stages of the disease. In addition, multivariate Cox proportional hazard survival analyses indicated that high RRM2 expression levels were an independent prognostic factor in patients with NPC. Furthermore, patient age, T stage and UICC stage were independent predictive factors for OS, which is consistent with previous studies $(12,15)$, and suggests that RRM2 expression may serve as a novel prognostic biomarker for NPC.

RRM2 overexpression led to an increase in cell migration and in cell invasion ability compared with control cells (Fig. 4C and D), suggesting that RRM2 may contribute to NPC cell metastasis. Furthermore, a high RRM2 expression level was associated with metastasis. However, the correlation was not significant, which may have been due to the limited sample size.

Although the clinical significance of RRM2 in a number of cancers has been established, the mechanism underlying the involvement of RRM2 in human cancers is complex (33-35). RRM2 expression may be regulated by the upstream kirsten rat sarcoma viral oncogene homolog and studies have shown that it may enhance the proliferation ability of cancer cells (33). Recently, Zhang et al (34) reported that RRM2 overexpression downregulates thrombospondin-1 expression in oropharyngeal cancer cells and upregulates vascular endothelial growth factor expression, which promotes the formation of tumor blood vessels. Duxbury et al (35) found that RRM2 expression activates the pancreatic cancer cell matrix metalloproteinases 9 via the $N F-\kappa B$ signaling pathway, which enhances the invasive ability of cancer cells. These studies therefore further support the hypothesis that RRM2 affects the proliferation, invasion and metastatic capabilities of malignant tumor cells.

In the present study, RRM2 overexpression led to an increase in NPC cell proliferation, migration and invasion capability, indicating that RRM2 may serve as a target for NPC treatment. Furthermore, studies have shown that inhibiting RRM2 expression greatly reduces neoplastic transformation in vivo (36) and increases the sensitivity of pancreatic cancer cells to chemotherapy using gemcitabine (37). Using small interfering RNA to target RRM2 nanoparticles led to inhibition of head and neck tumor cell growth in nude mice in vivo (38). 
In conclusion, the present study investigated the role of RRM2 expression in NPC, and demonstrated a high level of expression of RRM2 to be associated with metastasis and survival in patients with NPC. RRM2 expression was an independent predictive factor for DMFS and OS in patients with NPC. RRM2 overexpression also promoted NPC cell growth, migration and invasion. These results therefore suggest that RRM2 may be a potential biomarker for controlling metastasis and for improving prognostication in patients with NPC, and may provide a basis for the development of potential gene therapy for this disease.

\section{Acknowledgements}

This study was supported by grants from the National Natural Science Foundation of China (grant nos. 81272950 and 81025014) and Guangdong Natural Science Foundation (grant no. S2012010009698).

\section{References}

1. Yu MC and Yuan JM: Epidemiology of nasopharyngeal carcinoma. Semin Cancer Biol 12: 421-429, 2002.

2. Edge S, Byrd DR, Compton CC, et al (Eds.): AJCC Cancer Staging Manual. 7th edition. Lippincott-Raven, Philidelphia, PA, 2009.

3. Chua DT, Ma J, Sham JS, et al: Improvement of survival after addition of induction chemotherapy to radiotherapy in patients with early-stage nasopharyngeal carcinoma: Subgroup analysis of two Phase III trials. Int J Radia Oncol Biol Phys 65: 1300-1306, 2006.

4. Tao Q and Chan AT: Nasopharyngeal carcinoma: molecular pathogenesis and therapeutic developments. Exper Rev Mol Med 9: $1-24,2007$

5. Tatsumi-Tamori A, Yoshizaki T, Miwa T and Furukawa M: Clinical evaluation of staging system for nasopharyngeal carcinoma: comparison of fourth and fifth editions of UICC TNM classification. Ann Otol Rhinol Laryngol 109: 1125-1129, 2000.

6. Patel SG and Shah JP: TNM staging of cancers of the head and neck: striving for uniformity among diversity. CA Cancer J Clin 55: 242-258; quiz 261-242, 264, 2005.

7. Liang FY, Sun W, Han P, Lu X, Lian YN and Huang XM: Detecting plasma Epstein-Barr virus DNA to diagnose postradiation nasopharyngeal skull base lesions in nasopharyngeal carcinoma patients: a prospective study. Chin J Cancer 31: 142-149, 2012.

8. Tang LQ, Chen QY, Fan W, et al: Prospective study of tailoring whole-body dual-modality [18F]fluorodeoxyglucose positron emission tomography/computed tomography with plasma Epstein-Barr virus DNA for detecting distant metastasis in endemic nasopharyngeal carcinoma at initial staging. J Clin Oncol 31: 2861-2869, 2013.

9. Cory JG and Sato A: Regulation of ribonucleotide reductase activity in mammalian cells. Mol Cell Biochem 53-54: 257-266, 1983.

10. Thelander M, Gräslund A and Thelander L: Subunit M2 of mammalian ribonucleotide reductase. Characterization of a homogeneous protein isolated from M2-overproducing mouse cells. J Bio Chem 260: 2737-2741, 1985.

11. Eriksson S and Martin DW Jr.: Ribonucleotide reductase in cultured mouse lymphoma cells. Cell cycle-dependent variation in the activity of subunit protein M2.J Biol Chem 256: 9436-9440, 1981.

12. Morikawa T, Maeda D, Kume H, Homma Y and Fukayama M: Ribonucleotide reductase M2 subunit is a novel diagnostic marker and a potential therapeutic target in bladder cancer. Histopathology 57: 885-892, 2010.

13. Kolesar J, Huang W, Eickhoff J, et al: Evaluation of mRNA by Q-RTPCR and protein expression by AQUA of the M2 subunit of ribonucleotide reductase (RRM2) in human tumors. Cancer Chemother Pharmacol 64: 79-86, 2009.

14. Zuckerman JE, Hsueh T, Koya RC, Davis ME and Ribas A: siRNA knockdown of ribonucleotide reductase inhibits melanoma cell line proliferation alone or synergistically with temozolomide. J Invest Dermatol 131: 453-460, 2011.

15. Morikawa T, Hino R, Uozaki H, et al: Expression of ribonucleotide reductase M2 subunit in gastric cancer and effects of RRM2 inhibition in vitro. Hum Pathol 41: 1742-1748, 2010.
16. Burton TR, Kashour T, Wright JA and Amara FM: Cellular signaling pathways affect the function of ribonucleotide reductase mRNA binding proteins: mRNA stabilization, drug resistance, and malignancy (Review). Int J Oncol 22: 21-31, 2003.

17. Song LB, Zeng MS, Liao WT, et al: Bmi-1 is a novel molecular marker of nasopharyngeal carcinoma progression and immortalizes primary human nasopharyngeal epithelial cells. Cancer Res 66: 6225-6232, 2006.

18. Song LB, Li J, Liao WT, et al: The polycomb group protein Bmi-1 represses the tumor suppressor PTEN and induces epithelial-mesenchymal transition in human nasopharyngeal epithelial cells. J Clin Invest 119: 3626-3636, 2009.

19. Liao WT, Song LB, Zhang HZ, et al: Centromere protein H is a novel prognostic marker for nasopharyngeal carcinoma progression and overall patient survival. Clin Cancer Res 13: 508-514, 2007.

20. Sun W, Guo MM, Han P, et al: Id-1 and the p65 subunit of $\mathrm{NF}-\kappa \mathrm{B}$ promote migration of nasopharyngeal carcinoma cells and are correlated with poor prognosis. Carcinogenesis 33: 810-817, 2012.

21. Wang HY, Sun BY, Zhu ZH, et al: Eight-signature classifier for prediction of nasopharyngeal [corrected] carcinoma survival. J Clin Oncol 29: 4516-4525, 2011.

22. Zhang YL, Li J, Mo HY, et al: Different subsets of tumor infiltrating lymphocytes correlate with NPC progression in different ways. Mol Cancer 9: 4, 2010.

23. Guigay J, Temam S, Bourhis J, Pignon JP and Armand JP: Nasopharyngeal carcinoma and therapeutic management: the place of chemotherapy. Ann Oncol 17 (Suppl 10): x304-x307,2006.

24. Ma BB and Chan AT: Recent perspectives in the role of chemotherapy in the management of advanced nasopharyngeal carcinoma. Cancer 103: 22-31, 2005.

25. Chen QY, Wen YF, Guo L, et al: Concurrent chemoradiotherapy vs radiotherapy alone in stage II nasopharyngeal carcinoma: phase III randomized trial. J Natl Cancer Inst 103: 1761-1770, 2011.

26. Au JS, Law CK, Foo W and Lau WH: In-depth evaluation of the AJCC/UICC 1997 staging system of nasopharyngeal carcinoma: prognostic homogeneity and proposed refinements. Int J Radiat Oncol Biol Phys 56: 413-426, 2003.

27. Mao YP, Xie FY, Liu LZ, et al: Re-evaluation of 6th edition of AJCC staging system for nasopharyngeal carcinoma and proposed improvement based on magnetic resonance imaging. Int J Radiat Oncol Biol Phys 73: 1326-1334, 2009.

28. Gautam A, Li ZR and Bepler G: RRM1-induced metastasis suppression through PTEN-regulated pathways. Oncogene 22: 2135-2142, 2003.

29. Cao MY, Lee Y, Feng NP, et al: Adenovirus-mediated ribonucleotide reductase R1 gene therapy of human colon adenocarcinoma. Clin Cancer Res 9: 4553-4561, 2003.

30. Liu X, Zhou B, Xue L, et al: Metastasis-suppressing potential of ribonucleotide reductase small subunit $\mathrm{p} 53 \mathrm{R} 2$ in human cancer cells. Clin Cancer Res 12: 6337-6344, 2006.

31. Filatov D, Björklund S, Johansson E and Thelander L: Induction of the mouse ribonucleotide reductase $\mathrm{R} 1$ and $\mathrm{R} 2$ genes in response to DNA damage by UV light. J Biol Chem 271: 23698-23704, 1996.

32. Engström Y, Eriksson S, Jildevik I, Skog S, Thelander L and Tribukait B: Cell cycle-dependent expression of mammalian ribonucleotide reductase. Differential regulation of the two subunits. J Biol Chem 260: 9114-9116, 1985.

33. Yoshida Y, Tsunoda T, Doi K, et al: KRAS-mediated upregulation of RRM2 expression is essential for the proliferation of colorectal cancer cell lines. Anticancer Res 31: 2535-2539, 2011.

34. Zhang K, Hu S, Wu J, et al: Overexpression of RRM2 decreases thrombspondin-1 and increases VEGF production in human cancer cells in vitro and in vivo: implication of RRM2 in angiogenesis. Mol Cancer 8: 11, 2009.

35. Duxbury MS and Whang EE: RRM2 induces NF-kappaB-dependent MMP-9 activation and enhances cellular invasiveness. Biochem Biophys Res Commun 354: 190-196, 2007.

36. Liu X, Zhou B, Xue L, et al: Ribonucleotide reductase subunits M2 and p53R2 are potential biomarkers for metastasis of colon cancer. Clin Colorectal Cancer 6: 374-381, 2007.

37. Duxbury MS, Ito H, Zinner MJ, Ashley SW and Whang EE: RNA interference targeting the M2 subunit of ribonucleotide reductase enhances pancreatic adenocarcinoma chemosensitivity to gemcitabine. Oncogene 23: 1539-1548, 2004.

38. Rahman MA, Amin AR, Wang X, et al: Systemic delivery of siRNA nanoparticles targeting RRM2 suppresses head and neck tumor growth. J Control Release 159: 384-392, 2012. 